\title{
Falls Prediction in Care Homes Using Mobile App Data Collection
}

\author{
Ofir Dvir ${ }^{1}$, Paul Wolfson ${ }^{2}$, Laurence Lovat ${ }^{2}$, and Robert Moskovitch ${ }^{1}$ \\ 1 Software and Information Systems Engineering, Ben Gurion University, Israel \\ 2 Interventional Surgical Sciences, University College London, United Kingdom \\ ofirdvi@post.bgu.ac.il p.wolfson@ucl.ac.uk l.lovat@ucl.ac.uk \\ robertmo@bgu.ac.il
}

\begin{abstract}
Falls are one of the leading causes of unintentional injury related deaths in older adults. Although, falls among elderly is a well documented phenomena; falls of care homes' residents was under-researched, mainly due to the lack of documented data. In this study, we use data from over 1,769 care homes and 68,200 residents across the UK, which is based on carers who routinely documented the residents' activities, using the Mobile Care Monitoring mobile app over three years. This study focuses on predicting the first fall of elderly living in care homes a week ahead. We intend to predict continuously based on a time window of the last weeks. Due to the intrinsic longitudinal nature of the data and its heterogeneity, we employ the use of Temporal Abstraction and Time Intervals Related Patterns discovery, which are used as features for classification. We had designed an experiment that reflects real-life conditions to evaluate the framework. Using four weeks of observation time window performed best.
\end{abstract}

Keywords: Temporal Data Mining · Outcomes Prediction · Falls Prediction.

\section{Introduction}

The ageing population portion in the society has grown rapidly and becomes a major challenge in healthcare worldwide. It is estimated that, by 2025, this group will number approximately 1.2 billion and expand to 2 billion by $2050{ }^{1}$. More than a third of the population above 65 years old fall each year. Approximately 1 in 10 falls results in a serious injury, such as hip fracture, major soft-tissue injury and head injury. The mortality rate following a fall, increases dramatically with the age, exceeding to 70 percent of accidental deaths in adults above 75 years old. Therefore, predicting fall risk will allow ideally prevention or interventions, which potentially reduce fall occurrences and fall-related costs [1]. Most studies, if not all, till now in falls prediction focused on hospital or nursing environments, consisting on demographic descriptives and data from electronic health records,

\footnotetext{
${ }^{1}$ World Health Organization and others:Active ageing, A policy framework. World
} Health Organization. Geneva, (2002) 
and not based on continuously documented data in care homes - as we do in this paper.Thus, typically existing studies focused on the characterization of risk profiles, based on broad demographics and the adults conditions, rather than their daily data. Numerous parameters associated associated with the risk of falling have already been identified $[2,3]$, such as physical characteristics or their medical history. Although, previous studies had reported models that predict falls in elderly [4] they typically used heterogeneous samples that included prior fall events. While falls history is the strongest single indicator and the most frequently used factor for fall prediction, it cannot be used in the identification of individuals at risk of falling for the first time - which we investigate in this study. Therefore, there is still a need for assessment tools that can predict the risk of a first fall onset. Nevertheless, most of these studies were limited by their use of summary metrics, and relying solely on data collected infrequently, rather than considering longitudinal data, such as risk factors that change over time [5]. This happened, since there was no data typically available of the adults daily routine, which makes our database exceptional in the opportunities it provides. In this study we explore for the first time the ability to predict falls in care homes, based on careres documentation through a mobile app. We use data from over 1,769 care homes and 68,200 residents across the UK, routinely collected by the Mobile Care Monitoring (MCM) over three years. The goal is to predict a fall a week ahead, based on a sliding observation time window continuously. Due to the nature of the longitudinal data, we extend in this study the use the Maitreya framework [16] to perform prediction using a case-crossover-control evaluation design to reflect real life conditions. The contributions of the paper are the following:

- A rigorous evaluation of the falls prediction on a novel real life large database

- Investigating for the first time First Fall Prediction, based on secondary use of care homes daily documentation based on a mobile app

- A comprehensive framework for falls prediction based on TIRPs extracted from a sliding window

\section{Background}

\subsection{Falls Risk Assessment}

According to the World Health Organization, approximately one third of the population over the age of 70 will fall and the likelihood rises with the age and frailty. Falls account for more than half of injury-related hospital admissions and 40 percentage of injury-related deaths in the elderly ${ }^{2}$. In addition to the human cost of falling (distress, pain, fractures, loss of confidence and loss of independence), fall pose a substantial financial burden on healthcare systems

\footnotetext{
${ }^{2}$ World Health Organization and World Health Organization. Ageing and Life Course Unit: WHO global report on falls prevention in older age. World Health Organization. (2008)
} 
and estimated to cost the NHS (National Health Care) more than $£ 2.3$ billion per year. Therefore falling has an impact on quality of life, health and healthcare costs [7]. Identifying care home residents risk for falls can facilitate targeted prevention [8], potentially reducing incidence and associated costs.

\subsection{Temporal Abstraction and TIRPs Mining}

In order to analyze heterogeneous multivariate temporal data, it was proposed to use Temporal Abstraction in order to transform the various variables into a uniform representation of symbolic time intervals, which enables later to perform time intervals analytics [6]. In this study we used only state temporal abstraction, in which values are being discretized into states based on given cutoffs, which are later being concatenated, when adjacent and having the same symbol, into symbolic time intervals. There are several relevant discretization methods including, Equal Width Discretization (EWD), in which the cutoffs are determined by dividing the values range into equal bins; Symbolic Aggregate approXimation (SAX) [9], which consists on the gaussian distribution of the data, and the cutoffs are derived from its mean and standard deviation; Temporal Discretization for Classification (TD4C), which is a supervised temporal discretization method that searches for cutoffs that result in the states having the most different distribution between the classes(TD4C) [10] and more. Previous studies had shown the advantages of TD4C in comparison to EWD and SAX. Once the data is in a uniform format of symbolic time intervals, TIRPs can be discovered and used as features for classification. To discover TIRPs several time intervals mining methods were developed in the past two decades often using a subset of Allen's temporal relations, which often used to represent the temporal relations between pairs of time intervals in symbolic time intervals mining. There are seven temporal relations: before, meet, overlap, contain, starts, finishes, and equals. Therefore, a TIRP $\mathrm{P}$ is defined as $P=I S, R$, where $I S=I_{1}, I_{2}, . ., I_{k}$ is a set of $k$ symbolic time intervals ordered lexicographically and $\mathrm{R}$ defines all the temporal relations among each of the $\left(k^{2}-k\right) / 2$ pairs of symbolic time intervals in I. Several TIRPs mining methods were developed along the past two decades, in which the TIRP representation was improved, as well as the mining structures $[6,12]$.

\subsection{TIRPs Based Classification}

Outcomes prediction modelling while employing the analysis of the longitudinal data is one of the most important and challenging research fields in medical data. The use of frequent patterns as features for classification and prediction of data and specifically in multivariate temporal data is increasingly reported in the past decades [11]. It is based on the idea that richer predictive information is in the temporal order of the data, rather than independent features. Interestingly, several studies proposed using TIRPs as features for classifying multivariate time series quite simultaneously [12]. A recent study introduced the Maitreya framework for the classification of multivariate time series via TIRPs [16], which 
is used in this paper. The Maitreya framework provides novel TIRP metrics for classification, representing the TIRPs' number of instances, and their average duration, in addition to binary, as well as an efficient TIRPs detection method called SingleKarmaLego [6].

\section{Methods}

We introduce here the TIRPs based falls prediction, starting with the data creation, and proceed with the falls prediction framework.

\subsection{Mobile App Data Collection}

In many countries, such as the United Kingdom, documenting care home's residents is mandatory, but despite technological advances the data collection process today, commonly, is still written manually on paperwork. Alternatively, our study consists on data that was collected from elderly using the Mobile Care Monitoring (MCM) mobile app. This mobile app enables a care home carers to continuously record residents' activities and statuses. Documentation may include from fluid consumption and medication administration, to exercise activities and mental stimulation, recorded continuously using a simple and intuitive interface (Figure 1). The system is icon driven with limited need in typing to insert information, designed to be suitable for non-native English speakers. The data is automatically uploaded to a central database, integrated to provide a comprehensive, detailed, up-to-date data available for the careres, residents' families, which allows later secondary analytics, which is demonstrated in this paper. Using the Care App, data about the residents are recorded in real-time rather than at the end of the shift, or a day. Every day, 1,769 care homes around the UK use the MCM app to document more than 2.4 million care notes over 68,200 residents.
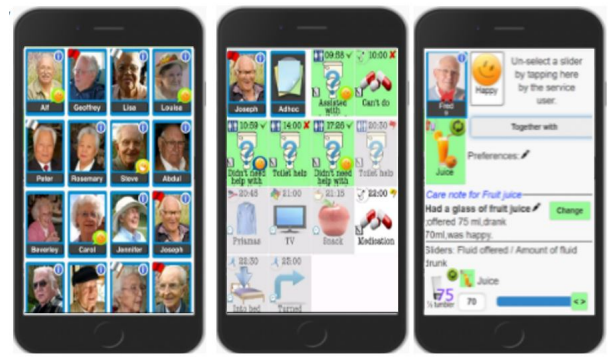

Fig. 1. Data collection using the icon driven mobile app.

\subsection{Problem Definition and Study Evaluation Design}

Given a database of documented data from the mobile app, the goal was to learn predictive models, based on a recent observation time window (of several weeks) 
that continuously provide a risk assessment a prediction time ahead (a week in this study). For that we create a cohort of cases of residents who had a fall in their records, and a group of matched controls, who are residents without a fall in their records. The controls are matched based on their age, gender and Care Quality Commission (CQC) score set by the independent regulator of health and social care in England. However, in order to create the prediction model which is an induced classifier, the observation time windows have to be labeled, which is illustrated in figure 2 .

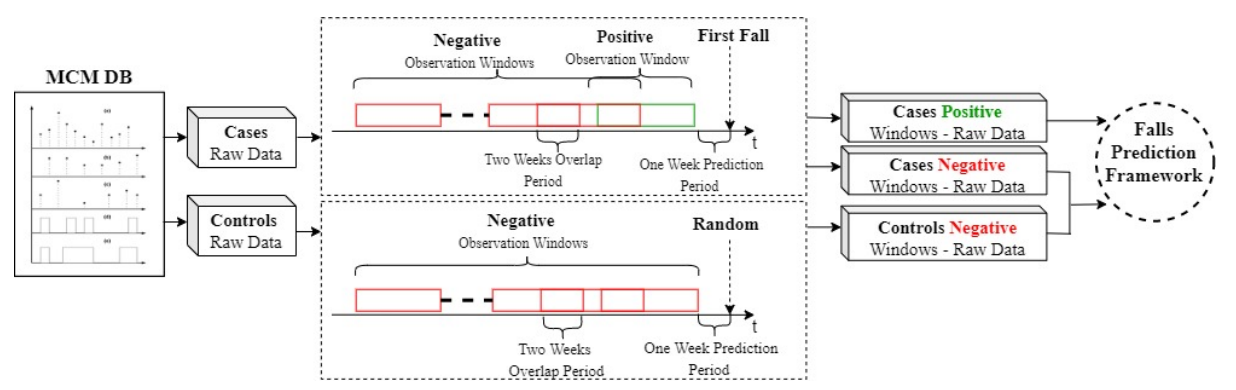

Fig. 2. Labeled observation time windows creation. From the MCM longitudinal database, the cases (residents who fell) are extracted, and their matched controls. Given the first fall in the cases' records, an observation time window is sled, in which the time window a week before the fall in each case is labeled as positive, and the earlier time windows are labeled as negative. In addition, random observation time windows from each matched control are labeled as negative, which enables a case-crossover-control design, reflecting real application conditions.

Thus, in each case the last observation time window prior to the prediction time period, which is just before the outcome event (which leaves potentially enough time for prevention) is labeled as positive. The negative time windows are taken from both the cases' earlier observation time windows, and from the controls' random observation time windows (since they do not have an outcome in their records). This corresponds to a case-crossover-control study design, which reflects real life conditions that are evaluated both on residents having a fall in their data, or not.

\subsection{FallPry}

We introduce FallPry for falls prediction, based on the MCM longitudinal database, that includes the time windows creation and their labeling, as was shown in figure 2, and a TIRPs based prediction framework that extends the use of the Maitreya framework [16]. The Maitreya framework includes four main components: Temporal Abstraction, Time Intervals Mining, TIRP-based Feature Representation, and Classifier induction. We explain here each of the framework components. 
Temporal Abstraction. The input raw data includes multiple variables that are described by time-point values series, or event (i.e., measurement, actions or risk assessment). Therefore, after dividing the raw data into positive and negative time windows the raw data being abstracted into a uniform format of symbolic time intervals, based on the use of state abstraction [10]. In this study we employed Equal Width Discretization (EWD); Symbolic Aggregate Approximation (SAX) [9] which creates states based on the gaussian distribution of the values; and Temporal Discretization for Classification (TD4C) [10], which is a supervised approach that determines the cutoffs for the states that increase the divergence of the classes values distribution.

TIRPs Discovery. After the variables went through temporal abstraction and are represented by symbolic time intervals, TIRPs can be discovered, which is done by the KarmaLego algorithm [12]. As mentioned in the background, we use non ambiguous TIRPs that use Allen's temporal relations to represent the relations between each pairwise symbolic time intervals. The discovered frequent TIRPs, which are referred as a Bag-of-TIRPs, are used as features for the classifier.

TIRPs based Classification. For that a features-matrix is constructed, in which the rows are the observation time windows, and the columns are the TIRPs (each time window is represented by a vector of values for each TIRPfeature, and the class). The TIRP-features' values are calculated according to the chosen TIRP representation metric: Boolean (whether the TIRP was detected in the time window), Horizontal Support (how many instances of the TIRP were detected), and Mean Duration (what was their average duration) [12]. Eventually, a classifier is induced from the training feature matrix. After a classifier was induced, given a new time window, the TIRPs are detected using the SingleKarmaLego algorithm [6] which results in a corresponding features vector which is given to the classifier to perform classification.

\section{Evaluation and Results}

We define here the research questions for the study, and its corresponding experimental plan and results. The experiment focused on examining the ability to predict first falls in care homes, based on the data from the mobile app entered by the careres.

\subsection{Research Questions}

1. How accurate can first fall be predicted a week ahead, and what is the best observation time window size?

2. What are the best FallPry settings for falls prediction? (i.e., state abstraction method, bins number, TIRP metric and classifier) 


\subsection{Data}

The data for this study were routinely collected from over ,1769 care homes across the UK over three years, from 2017 to 2019 by the icon-driven Mobile Care Monitoring (MCM) system from Person Centered Software, Guildford, UK (subsection 4.1). The total population includes 68,200 residents, of whom 13,412 are above 75 years old, not bed bound and had at least one full year of documentation. We define two groups of residents: the fallers and the non-fallers, which will be referred as the cases and controls group respectively. The non-fallers (control) were residents who have not had a documented fall during the entire documentation period while the fallers (case) are residents who had documentation of a fall with an injury of some severity level (in this study no separation was made between serious and moderate injury). Moreover, since this study focused on predicting residents' first fall, the fallers group was defined so throughout the entire period prior to the fall (at least six months) no falls were documented.

Table 1. Cases and Controls Statistics

\begin{tabular}{|l|l|l|l|}
\hline & All Residents & Case & Control \\
\hline $\mathrm{N}$ & 13,412 & 4894 & 8518 \\
Age(std) & $87.3( \pm 6.5)$ & $88( \pm 6.1)$ & $86.9( \pm 6.7)$ \\
Female(\%) & $71.2 \%$ & $73.9 \%$ & $69.7 \%$ \\
Female age, mean(std) & $87.8( \pm 6.4)$ & $88.4( \pm 6)$ & $87.8( \pm 6.4)$ \\
Male age, mean(std) & $85.9( \pm 6.5)$ & $86.8( \pm 5.9)$ & $85.9( \pm 6.5)$ \\
BMI under 18.5(\%) & $11.9 \%$ & $13 \%$ & $10.8 \%$ \\
\hline
\end{tabular}

\subsection{Experimental Setup}

We used the Maitreya framework (subsection 3.2) for all the experiments, having the following settings: a $50 \%$ minimal vertical support threshold, maximal gap of 7 days and the full set of Allen's seven temporal relations. In the experiments, we tested four discretization methods: EWD, SAX, TD4C with Kullback Leibler (TD4C-KL), and TD4C with cosine as the distance measure function (TD4C-Cosine). The number of states determines the level of granularity of the abstraction method, which we experimented with two, three, and four states. Three different TIRP metrics were used: binary (BIN), horizontal support (HS) and mean duration (MeanD) and four types of classifiers: K-Nearest Neighbor (KNN), XGBoost (XGB), Logistic Regression (LR) and Neural Net (NN) using the default settings of sklearn. We ran the experiments using 10-fold crossvalidation, and using AUC as the metric to evaluate the prediction performance. To evaluate our models in conditions that reflect the real application of a potential time-dependent falls prediction model, we train and test our model by positive and negative time windows taken from the case and control groups defined above (subsection 3.2). The positive and negative time windows were selected in ration of 1:6. 


\subsection{Falls Prediction Framework Baseline - Features Based Approach}

As an alternative to the TIRPs based prediction, we implemented a feature based approach, in which features were extracted from each time window. Data aggregation has been widely applied as effective techniques to reduce data redundancy and simplify complex data, especially in longitudinal data[13,14]. Due to the heterogeneity of the various variables, and sparseness we decided to perform Piecewise Aggregate Approximation [15], based on ten time periods, for which the mean is calculated, as done in PAA, which will become the features. Although in PAA only the mean is calculated, we used count for specific variables that contain events, such as the exercises frequency, nutritional risk assessment frequency and more per observation time period. Eventually these ten time periods representative values become features. Performing the aggregation on each time window individually, rather than preforming it on the whole time window's data which enables to capture the temporal relations to yield meaningful results.

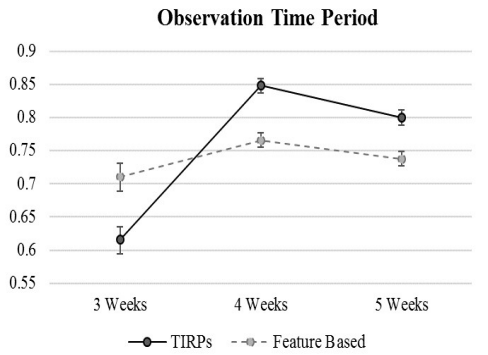

Fig. 3. Using FallPry preform bather then the feature based approach when using both 4 and 5 weeks observation time window

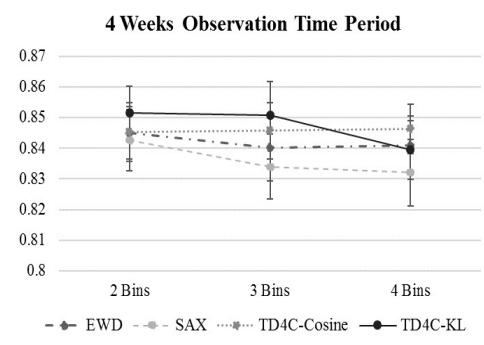

Fig. 4. The use of TD4C KL with two bins (states) performed best using 4 weeks observation time period.

\subsection{Results}

Experiment - Elderly First Injury Fall Prediction In this experiment the performance in predicting the first fall with a prediction time of one week ahead was evaluated (research question A). For the observation time periods, three, four or five weeks were used. Additionally, we compared the combinations of the state abstraction methods, bins number, and TIRP representation metrics, with the four different classifiers (research question B). As a baseline, the feature based approach (subsection 4.5) was used that were extracted from the same time windows. Figure 4 shows the mean results, including $95 \%$ confidence intervals, for each observation time period of 3, 4, or 5 weeks, while using FallPry with TIRPs or Features for comparison. The use of the TIRPs based was better with 4 or 5 weeks, while the Features based was better with 3 weeks. However, 4 weeks was significantly better with the use of TIRPs. In figure 5, the use of FallPry 
with TIRPs is presented with the various state abstraction methods (EWD, and the number of states $(2,3$, or 4 bins), in which the TD4C-KL seem to outperform, especially with 2 or 3 bins. Figure 6 , shows the mean results for the TIRPs based, including the various TIRPs' metrics of Binary (BIN), Horizontal Support (HS) and Mean Duration (MD), and the Features based, with the four types of classifiers that were used. There is a chart for each of the observation time periods. It can be seen that the 4 weeks has the best performance, especially with the XGB classifier and mean duration (which describes the average duration of the TIRP's instances). Thus, using Maitreya with 4 weeks observation time window, 2 bins, TD4C-KL discretization, MD representation and XGB model achieves the best performance (88.3\% AUC, question B)

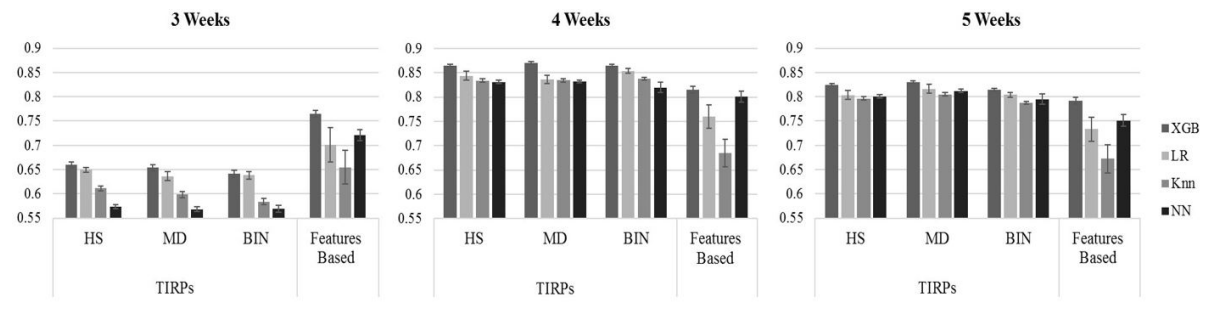

Fig. 5. FallPry preform better then the feature based approach when using both 4 and 5 weeks observation time windows. The XGB was significantly better than the KNN, $\mathrm{NN}$ and LR in all cases.

\section{Discussion and Conclusions}

Falls in elderly population is one of the leading causes for rapid deterioration and death. Being able to predict a fall, or assess the risk for a fall, is desirable in order to increase caution and ideally decrease the risk. Most of the literature so far that reported the intention to predict falls was in hospital environments, consisting on Electronic Health Records data, or in nursing homes, consisting on data collected infrequently and manually [5]. In this study, for the first time, as far as we know, falls prediction in care homes is performed based on such rich daily data of residents that was documented through a mobile app by the care homes' careres. Moreover, our datasets include residents' daily activities, nutrition and medical status which routinely documented from over 1,769 care homes and 68,200 residents. An experiment, reflecting real-life conditions, was performed that focused on evaluating the falls prediction a week ahead, given a sliding observation time window of 3,4 or 5 weeks duration. Using 4 weeks observation time window performed significantly better than 3 or 5 weeks. Also, using the TD4CKL abstraction method with two states and the mean duration TIRP metric, with the XGB classifier achieved the highest performance. For future work we would like to reduce the number of variables, and understand better the predictors for the falls, as well as experiment with the frequency of the risk estimations. 


\section{References}

1. Rao, S.S.:Prevention of falls in older patients. American family physician, 72(1), pp.81-88 (2005)

2. Bloch, F., Thibaud, M., Tournoux-Facon, C., Brèque, C., Rigaud, A.S., Dugué, B. and Kemoun, G.: Estimation of the risk factors for falls in the elderly: Can meta-analysis provide a valid answer?. Geriatrics gerontology international, 13(2), pp.250-263 (2013).

3. Gillespie, L.D., Robertson, M.C., Gillespie, W.J., Sherrington, C., Gates, S., Clemson, L.M. and Lamb, S.E.: Interventions for preventing falls in older people living in the community. Cochrane database of systematic reviews,(9) (2012).

4. Kojima, G., Masud, T., Kendrick, D., Morris, R., Gawler, S., Treml, J. and Iliffe, S.: Does the timed up and go test predict future falls among British community-dwelling older people? Prospective cohort study nested within a randomised controlled trial. BMC geriatrics, 15(1), p.38 (2015).

5. Marier, A., Olsho, L.E., Rhodes, W. and Spector, W.D.: Improving prediction of fall risk among nursing home residents using electronic medical records. Journal of the American Medical Informatics Association, 23(2), pp.276-282 (2016).

6. Moskovitch, R. and Shahar, Y.: Classification of multivariate time series via temporal abstraction and time intervals mining. Knowledge and Information Systems, 45(1), pp.35-74 (2015).

7. Close, J.C.: Prevention of falls in older people. Disability and rehabilitation, 27(1819), pp.1061-1071 (2005).

8. Kamel, H.K.: Q A with the Expert on: Falls Preventing Falls in the Nursing Home. Annals of Long Term Care, 14(10), p.42 (2006).

9. Lin, J., Keogh, E., Lonardi, S. and Chiu, B.: June. A symbolic representation of time series, with implications for streaming algorithms. In Proceedings of the 8th ACM SIGMOD workshop on Research issues in data mining and knowledge discovery, pp. 2-11 (2003).

10. Moskovitch, R. and Shahar, Y.: Classification-driven temporal discretization of multivariate time series. Data Mining and Knowledge Discovery, 29(4), pp.871-913 (20015).

11. Batal, I., Valizadegan, H., Cooper, G.F. and Hauskrecht, M.: A pattern mining approach for classifying multivariate temporal data. In 2011 IEEE International Conference on Bioinformatics and Biomedicine, pp.358-365 (2011).

12. Moskovitch, R., Walsh, C., Wang, F., Hripcsak, G. and Tatonetti, N.: Outcomes prediction via time intervals related patterns. In 2015 IEEE international conference on data mining, pp. 919-924 (2015).

13. Lu, Y., Comsa, I.S., Kuonen, P. and Hirsbrunner, B.: Dynamic data aggregation protocol based on multiple objective tree in wireless sensor networks. In 2015 IEEE tenth international conference on intelligent sensors, sensor networks and information processing (ISSNIP), pp. 1-7 (2015).

14. Min, K. and Corso, J.J., 2019. TASED-net: Temporally-aggregating spatial encoder-decoder network for video saliency detection. In Proceedings of the IEEE International Conference on Computer Vision, pp. 2394-2403 (2019).

15. Keogh, E.J. and Pazzani, M.J.: August. Scaling up dynamic time warping for datamining applications. In Proceedings of the sixth ACM SIGKDD international conference on Knowledge discovery and data mining, pp. 285-289 (2000).

16. Moskovitch, R., Polubriaginof, F., Weiss, A., Ryan, P. and Tatonetti, N.: Procedure prediction from symbolic Electronic Health Records via time intervals analytics. Journal of Biomedical Informatics, 75, pp.70-82 (2017). 